\title{
In-flight calibration of the Cluster/CODIF sensor
}

\author{
L. M. Kistler ${ }^{1}$, C. G. Mouikis ${ }^{1}$, and K. J. Genestreti ${ }^{1, *}$ \\ ${ }^{1}$ University of New Hampshire, Durham, NH, USA \\ *now at: Southwest Research Institute, San Antonio, TX, USA \\ Correspondence to: L. M. Kistler (lynn.kistler@unh.edu)
}

Received: 2 April 2013 - Published in Geosci. Instrum. Method. Data Syst. Discuss.: 3 May 2013

Revised: 12 August 2013 - Accepted: 9 September 2013 - Published: 10 October 2013

\begin{abstract}
The Cluster/CODIF sensor is a time-of-flight instrument that measures the ion composition over the energy range $40 \mathrm{eV} \mathrm{e}^{-1}$ to $40 \mathrm{keV} \mathrm{e}^{-1}$. It operated for $4 \mathrm{yr}$ on $\mathrm{S} / \mathrm{C} 1$, $9 \mathrm{yr}$ on S/C 3, and is still operational on S/C 4, after more than 12 yr. During this time the total ion detection efficiency has decreased by a factor of 50. In this paper, we describe the methods used to track the efficiency changes throughout the mission for the three different spacecraft and for the different ion species. The methods include calculations of the efficiencies using rate data collected in the instrument, comparisons with other instruments on the Cluster satellites, and checks based on geophysically reasonable assumptions.
\end{abstract}

\section{Introduction}

The Cluster/CODIF instrument measures ion composition over the energy range $\sim 40 \mathrm{eV}$ to $40 \mathrm{keV} \mathrm{e}^{-1}$. The CODIF instrument operated on S/C 1 from 1 February 2001 to 24 October 2004, on S/C 3 from 1 February 2001 to 11 November 2009, and on S/C 4 from 1 February 2001 through the present time, and is still operating. The instrument never operated on S/C 2. The Cluster/CODIF instrument is a combination of an electrostatic analyzer followed by postacceleration of $15 \mathrm{kV}$, and then a time of flight section, as described in Rème et al. (2001). Details of the instrument are also discussed in Möbius et al. (1998). The entrance into the electrostatic analyzer is divided into two $180^{\circ}$ sections. Grids in the entrance define the geometric factor of the two sides. One side, with highly transparent grids, is called the "high side", or HS. The other side, with grids that drop the flux by a factor of 100, is called the "low side", or LS. Only one side is used at any time.
After exiting the analyzer, ions go through a thin carbon foil at the entrance to the time-of-flight section. Electrons knocked out of the foil are steered to a microchannel plate (MCP). The MCP pulse from the electrons is used to create two signals: a "start signal", which is used to initiate the time-of-flight calculation, and a "position" signal that indicates the direction from which the ion entered the instrument. The position is determined by detecting the signal in one of eight $22.5^{\circ}$ sections. After passing through the foil, the ion traverses the flight path and hits an MCP to create the "stop" pulse. A "valid event" within the instrument requires a "start" and a "stop" pulse, to get the time-of-flight, and a "position" pulse. The ion species (mass per charge) is determined by the combination of the energy per charge of the ion, from the electrostatic analyzer, and the time-of-flight of the ion over the known flight path length.

The number of ions detected for a given input flux depends on a number of factors. First, it is a function of the geometric factor, which includes the geometry of the electrostatic analyzer and the transparency of any grids in the optics path. Then it is determined by the efficiency of the three required signals. The start pulse efficiency depends on the number of electrons emitted from the carbon foil, which varies statistically with the particle energy and species (Ritzau and Baragiola, 1998; Allegrini et al., 2003), the fraction of the electrons that are steered to the detector, and the active area of the MCP itself $(\sim 50 \%)$. The start and position signals are obtained from the electron pulse from the MCP by a grid that separates the pulse into two parts. The start signal comes from the grid, while the position signal comes from the electrons that go through the grid to an anode (Fig. 11 in Rème et al., 2001). Thus the difference in efficiency of the detection of the two signals depends on the transparency of the grid, and on the thresholds in the two sets of electronics that detect 
the signals. The size of the pulse from the MCP has some statistical variation. If operating at optimum gain, each ion would give a pulse large enough to be measured. However if the gain is not optimum, some fraction of the signals will be below the detection threshold. The stop pulse efficiency depends on the fraction of ions that reach the detector, and again, the active area of the MCP and the electronics detection efficiency. The fraction of ions that reach the detector is a function of the amount that the ions scatter in the foil, which depends on the particle species and energy (e.g., Gonin et al., 1992). Finally, to be counted as a particular ion species, the ion must have a time-of-flight that falls between defined limits. Because of energy loss in the foil (e.g., Allegrini et al., 2006), the peaks in time of flight are not symmetric, but have a tail towards longer times of flight (Fig. 16 in Rème et al., 2001). Some fraction of the ions will fall outside the defined limits (Fig. 17 in Rème et al., 2001). This also needs to be taken into account.

Most of these dependencies are not expected to change with time. The instruments were well calibrated before launch over the full range of energies using the four major ion species expected in the instrument: $\mathrm{H}^{+}, \mathrm{O}^{+}, \mathrm{He}^{+}$, and $\mathrm{He}^{++}$. The results of the ground calibrations are summarized in Kistler (2000a,b,c). However, the gain of the microchannel plates does decrease significantly over time (Sandel et al., 1977; Drake et al., 1998; Kishimoto et al., 2006). In principle, the gain can be increased by increasing the MCP voltage. However there are two aspects of the instrument that limit the effectiveness of raising the voltage. The first aspect is the MCP geometry. The instrument uses large MCPs with the same MCP covering both the start and stop signals. In addition, the instrument has 4 quadrants of MCPs, covering the full $360^{\circ}$ entrance, both the LS and the HS, and there is only one MCP power supply for the four quadrants. Thus, while the efficiency may decrease on one part of an MCP, or on one set of MCPs, increasing the voltage will increase the gain over the whole MCP area. The second aspect is that the MCPs are located at high voltage (HV), and the electron pulse from the MCP goes across the high-voltage gap $(\sim 12 \mathrm{kV})$ to the anode plane (Fig. 11 in Rème et al., 2001). If the gain gets too high, there can be a large current across the high-voltage gap that can trigger the HV power supply to shut down. Since the gain of the MCPs does not change uniformly, part of an MCP may require a higher voltage, but that makes the gain too high in other areas. Thus there is a limit to how high the MCP voltage can be raised. While the MCP voltages have been increased to improve the gain over the course of the mission, the instrument has also had to operate with a reduced gain, and tracking this non-uniform gain change, and determining how it affects the efficiencies of the different species, is a significant calibration effort.

An overview of some of the in-flight calibration techniques used for Cluster/CODIF, and the resulting calibrations through 2003 are presented in McFadden et al. (2007). In this paper we provide an updated description of the calibration techniques, including additional cross-calibration techniques that have been applied later in the mission, and show how the calibrations have changed over the $12 \mathrm{yr}$ time period.

\section{$2 \mathbf{H}^{+}$calibration}

Because $\mathrm{H}^{+}$dominates the flux of ions almost all the time, $\mathrm{H}^{+}$can be calibrated using the widest range of techniques. The "engineering rate" counters, which count the start, stop, and position pulses, are dominated by $\mathrm{H}^{+}$. This allows the efficiency of $\mathrm{H}^{+}$to be determined directly. Geophysically reasonable assumptions can also be used to check the determined efficiency. The ion pressure is usually dominated by $\mathrm{H}^{+}$, so in some cases the $\mathrm{H}^{+}$calibration can be checked by monitoring for pressure balance as the spacecraft transitions through different regions in the magnetosphere. In addition, the ion and electron densities in a plasma should be the same, so in time periods where the CODIF energy range carries the dominant density, the CODIF-measured density can be compared with that measured by the electrons with the PEACE instrument (Johnston et al., 1997), with the electron density determined through wave measurements by the WHISPER instrument (Décréau et al., 2001; Trotignon et al., 2010), as well as with the ion density calculated by the all-ion instrument, Hot-Ion Analyzer (HIA; Rème et al., 2001).

\subsection{High side signal efficiencies using engineering rates}

A valid event in the CODIF instrument requires a start and a stop pulse in coincidence, and one and only one position signal. The efficiencies for getting a start pulse and a stop pulse can be determined using the start rate (SF), the stop rate (SR), and the start/stop coincidence rate (SFR). The fraction of ions that give a coincidence for each measured start count gives a measure of the stop efficiency. Similarly, the fraction of ions that give a coincidence for each measured stop count is a measure of the start efficiency. The fraction of the ions that give a single position, SEV, for each measured coincidence, SFR, is the "single position" efficiency. To summarize,

$$
\begin{aligned}
& \text { Stop_Efficiency }=\text { SFR } / \text { SF } \\
& \text { Start_Efficiency }=\text { SFR } / \text { SR } \\
& \text { Single_Position_Efficiency }=\text { SEV } / \text { SFR. }
\end{aligned}
$$

The product of these three efficiencies gives the total efficiency. Again, we note that these rates count all species, but are normally dominated by $\mathrm{H}^{+}$.

There is one final caveat that applies to the stop rate. While SF and SFR directly measure the signal used to start the timeof-flight and the coincidence rate, the SR is a separate signal derived from the stop rate, but is not directly the signal used by the time-of-flight circuit. Thus, the threshold on the SR signal may be different from the threshold of the "stop" signal used in the time of flight. While an effort was made to adjust the threshold prior to launch, the actual thresholds ended 
up different on the three operating sensors. S/C 1 had the SR that most reliably tracked the real stop, while the instruments on the other two spacecraft had thresholds set somewhat too high, so that in general, the SR is lower than the actual "stop" rate. As a result, calculations of the start efficiency using this method can be greater than 1 . When CODIF on S/C 1 operated, we used S/C 1 for our baseline calibration, and then verified the other two spacecraft calibrations through crosscalibration with S/C 1. After 2004, when CODIF on S/C 1 no longer operated, we began to rely on the pressure balance technique in the magnetotail and cusp as a method to crosscheck the calibration. This will be discussed in Sect. 2.2

Figure 1 shows the "stop", "start", and "single position" efficiencies for $1 \mathrm{keV}$ ions from 2001 into 2012 for the HS of CODIF on S/C 4. The bottom panel shows the MCP set values over the mission. Clearly, the sharp increases in efficiencies correspond with time periods when the MCP voltage was increased. The changes in the stop efficiency (top panel) are relatively modest. It started at $\sim 0.65$ at the beginning of mission, and dropped to 0.4 at its lowest, in 2008 and 2009. In July 2009 a final attempt to increase the voltage was performed, which brought the stop efficiency up to $\sim 0.5$, where it has remained, quite stable, since then. We believe that the relative stability of the stop pulse efficiency is due to the spread of ions that create the stop signal on the MCP. Because they are spread over a large area of the MCP, due to scattering in the foil, no single area of the MCP has had a large decrease in efficiency.

The start efficiency, shown in the second panel, has changed more dramatically. As noted above, the numbers greater than one are due to the thresholds of the SR. But the efficiency overall has dropped to $25 \%$ of its initial value. The final MCP increase brought it back up slightly, but it is still significantly lower than what it had been at the start of the mission. The greater decrease compared to the "stop" signal is because the electrons from the foil are steered and focused onto the MCP, towards the center of each position. Thus, only a small area of the MCP receives the majority of the flux resulting in a larger decrease in efficiency.

The single position efficiency (third panel) also began decreasing after launch. The reason for this was initially not clear. Because the position signals in general have a lower threshold than the start signals, any signal that gives a start should also give a position, even when the pulse height is small. Thus any start/stop coincidence (SFR) should have had a corresponding position signal, and the expected SEV / SFR ratio should be close to 1 . We found that the reason that the single position efficiency decreases is an error in the event logic. The registers that record that a position signal has been observed are not cleared at the beginning of an event. As a result, if an ion generates a "position" signal but not a start signal, that position is saved in the register, but not counted as an event. When a new event occurs that does have a start signal and a position signal, the logic checks whether one and only one position signal has been detected and finds
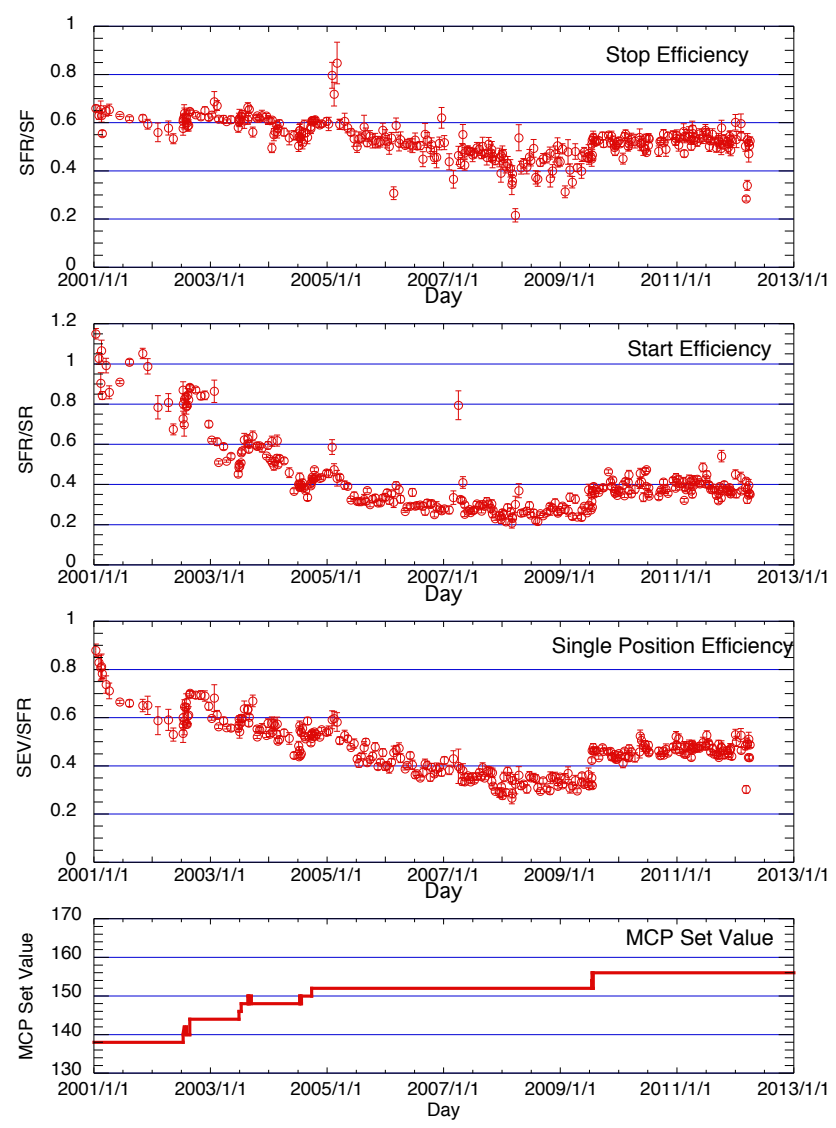

Fig. 1. Stop, start, and single position efficiencies for SC4 from 2001 to 2012 for $1 \mathrm{keV}$ ions. The bottom panel shows the setting for the voltage across the MCP.

two positions. Thus the event is rejected. At the beginning of the mission, almost all events that had a position signal also had a start signal. But as the MCP gain decreased, it increased the probability that a position signal would be measured with no corresponding start signal, which generates the invalid events. Thus, when the start efficiency goes down, the single position efficiency also goes down, essentially squaring the effect of the decrease in start efficiency in the total efficiency calculation.

\subsection{High side total efficiencies}

As discussed above, because of issues with the SR on S/C 4, the engineering rates give an indication of how the efficiencies have changed, but do not give the absolute value. Starting towards the end of 2004, when CODIF on S/C 1 stopped operating, we use pressure balance in the magnetotail and cusp to validate the changes in efficiency. Figure 2 shows an example illustrating the technique. In the magnetotail, the pressure changes from being dominated by the magnetic field close to the lobes to being dominated by the plasma pressure close to the neutral sheet, with the overall pressure 


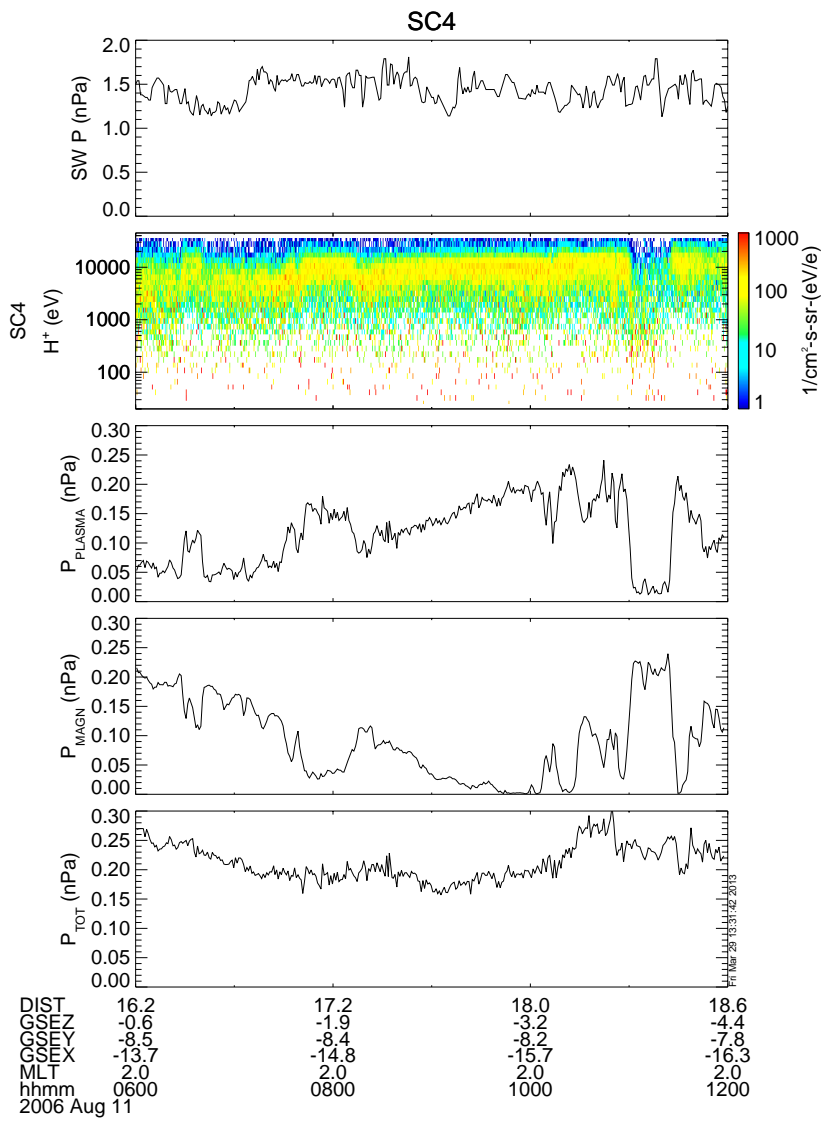

Fig. 2. An example time period when pressure balance in the tail can be used to check the $\mathrm{H}^{+}$calibration. The panels, from top to bottom, give the solar wind pressure, the $\mathrm{H}^{+}$energy spectrogram, the plasma pressure, the magnetic field pressure, and the total pressure.

remaining approximately constant (Fairfield et al., 1981). During a plasma sheet encounter, the tail flaps or waves, so that the spacecraft moves from areas dominated by magnetic pressure to areas dominated by particle pressure. In Fig. 2, the top panel shows the solar wind pressure at this time. This information is used in order to avoid time periods that have a large change in the solar wind pressure. The second panel shows the $\mathrm{H}^{+}$energy spectrum during the plasma sheet encounter. The next three panels show the plasma pressure, the magnetic field pressure, and the total pressure. In this example, the spacecraft starts close to the lobe, with the pressure dominated by the magnetic field. It then moves closer to the neutral sheet, reaching the neutral sheet at $\sim 10$ UT. During this time, the magnetic pressure in general decreases while the plasma pressure increases. However there are a number of brief excursions to regions of higher plasma pressure, notably at 06:30 and 07:45 UT. We have adjusted the CODIF efficiency by hand for each event so that the total pressure, shown in the last panel, maintains the smoothest possible profile during these sharp transitions.

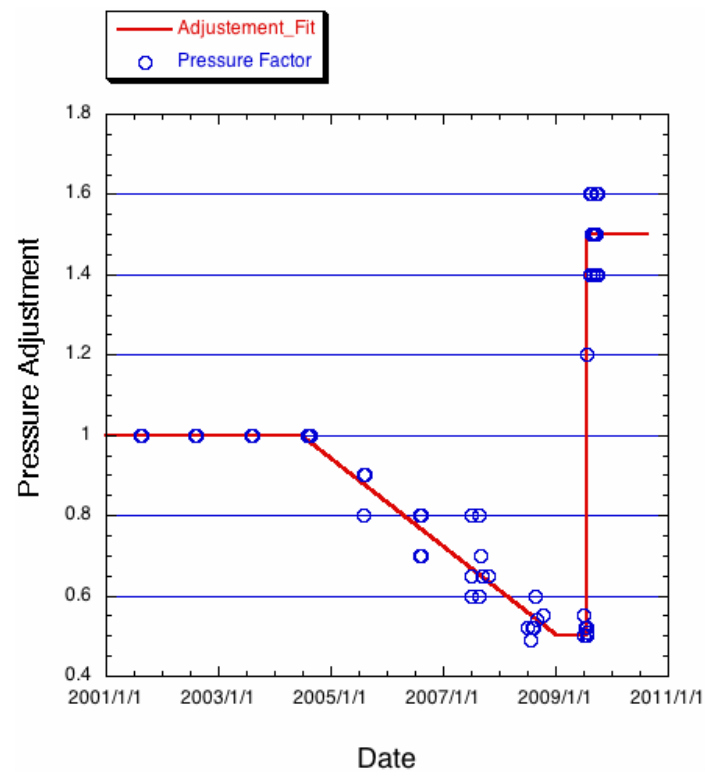

Fig. 3. Adjustment factors to the efficiencies, determined using pressure balance. The large increase corresponds to when the MCP voltage was increased in 2009.

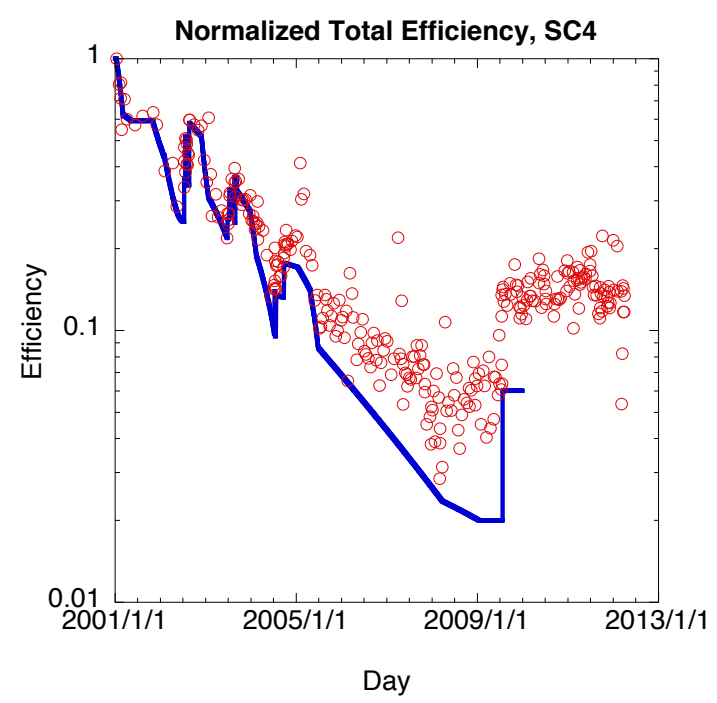

Fig. 4. Normalized total efficiencies derived using rate data (circles) and after the adjustments based on the pressure balance technique (blue line). The efficiencies are normalized to 1.0 at the start of mission.

Figure 3 shows the adjustment parameters that were determined for time periods after 2004, and the adjustment curve that was derived. This curve was used to modify the total efficiency determined from the rate data. The results are shown in Fig. 4. The red circles show the normalized total efficiency determined by taking the product of the three ratebased efficiencies shown in Fig. 1, and normalizing the result to 1.0 at the start of the mission. The blue line shows 


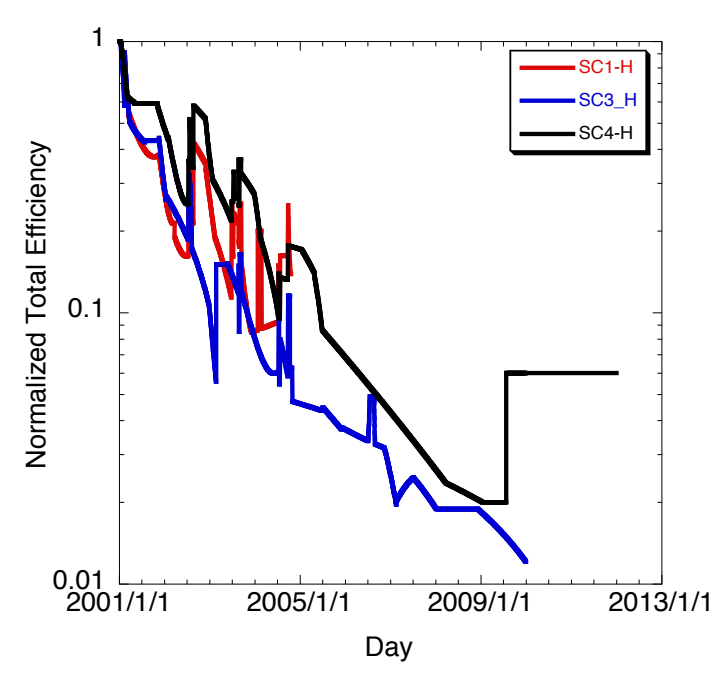

Fig. 5. $\mathrm{H}^{+}$normalized total efficiencies for the high side of the three CODIF instruments.

the final normalized total efficiency, determined by adjusting the curve determined from the rate data with the pressure adjustment curve from Fig. 3. Again, the reason that an adjustment is required is due to the inaccurate measure of the stop rate, $\mathrm{SR}$.

Figure 5 shows the $\mathrm{H}^{+}$normalized total efficiencies for the HS on all three instruments. The overall changes were similar on the three spacecraft.

\subsection{Low side total efficiencies}

As discussed in the introduction, the LS has a geometric factor that is a factor of 100 smaller than the HS. Thus the amount of flux on its MCPs is dramatically less. As a result, its efficiencies remained essentially constant for the first four years of the mission. However, after that, they also started to decline. The low side of the instrument is used predominantly on S/C 4 in the magnetosheath and in the solar wind. In these regions, the high flux saturates the electronics on the high side, and so the low side is used. In the magnetosheath, the WHISPER instrument is able to derive the total plasma density by identifying the plasma frequency in the data. The HIA all-ion instrument has been well cross-calibrated against WHISPER in this region (see Blagau et al., 2013). The dominant particle energy in the magnetosheath is $\sim 1 \mathrm{keV}$, so the distribution is well contained within the CODIF energy range, as well as in the energy ranges covered by HIA and PEACE. Thus it is fitting to also cross-calibrate the Cluster LS against these instruments.

The LS calibration has been done in two ways. First, the pressure measured by the CODIF instrument is compared before and after the time when CODIF switches from the HS to the LS. This normally occurs in the outer magnetosphere when the flux is low, and is dominated by high-energy $(\sim 10 \mathrm{keV})$ ions. Second, the CODIF densities are compared

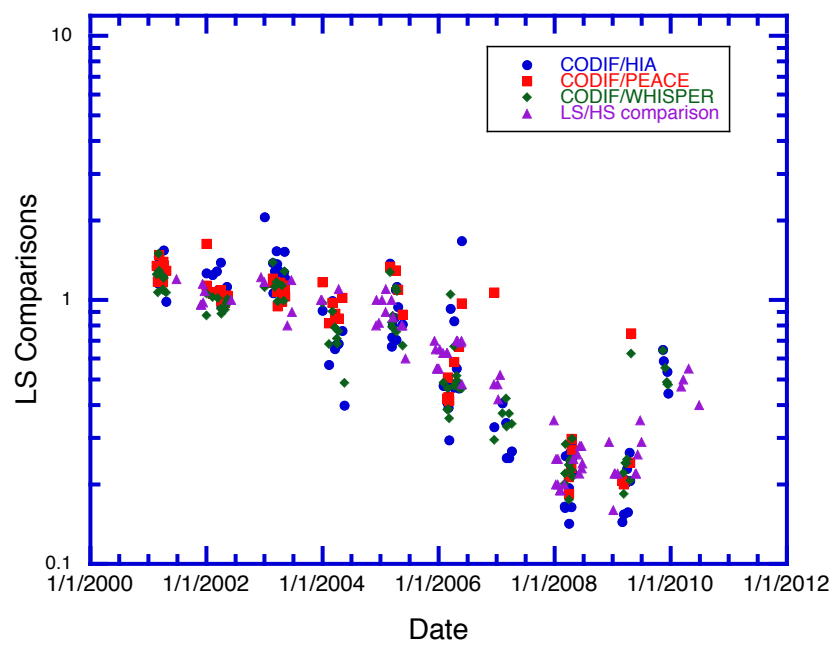

Fig. 6. CODIF SC/4 LS comparisons with the density measurements from HIA, PEACE (electrons) and WHISPER (electrons), and with pressure measurements from the CODIF HS.

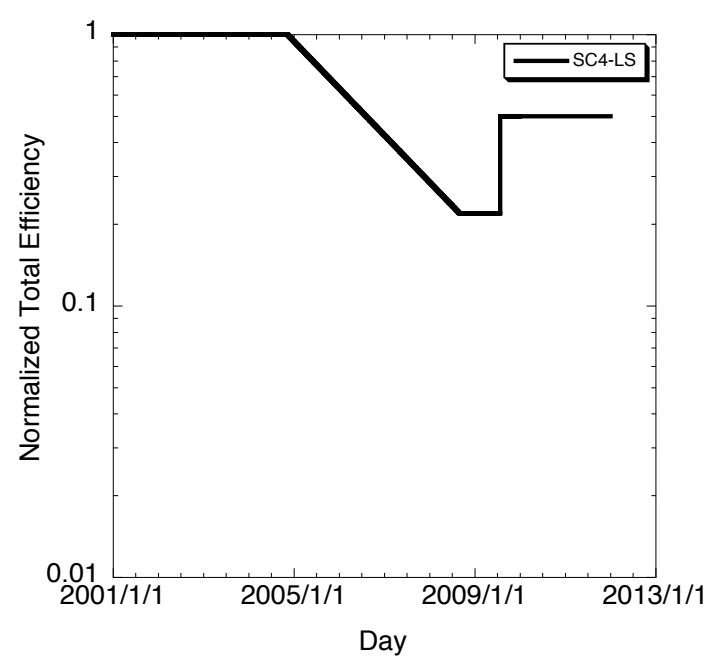

Fig. 7. $\mathrm{H}^{+}$normalized total efficiencies for the low side of SC 4 .

with electron densities from WHISPER and PEACE, and ion densities from HIA in the magnetosheath. All data were obtained from the Cluster Active Archive (CAA). In this case, the dominant energy is $\sim 1 \mathrm{keV}$. Figure 6 shows the ratio of the CODIF LS measurement with the density measurements by HIA, PEACE and WHISPER, and the pressure measurements using the HS of CODIF. The comparisons with the HS on CODIF were done after the HS efficiency corrections had been finalized. All methods consistently show that the LS side efficiency began decreasing in 2005. When the MCP voltage was increased in 2009 , the efficiency then increased, but only up to $50 \%$ of its initial value. From these measurements, the normalized total efficiency curve for the LS, shown in Fig. 7, was determined. 
a)

\begin{tabular}{|c|c|c|c|c|c|c|c|c|c|c|c|c|c|c|c|c|c|}
\hline \multirow[b]{2}{*}{32 Sweeps } & \multicolumn{5}{|r|}{$1 / 4 \mathrm{spin}$} & \multicolumn{4}{|r|}{$1 / 2 \mathrm{spin}$} & \multicolumn{4}{|r|}{$3 / 4$ spin } & \multicolumn{4}{|r|}{$1 \mathrm{spin}$} \\
\hline & 0 & 2 & 3 & 4 & 7 & 8 & $10 \quad 11$ & $12 \quad 13$ & $14 \quad 15$ & $16 \quad 17$ & $18 \quad 19$ & $20 \quad 21$ & $22 \quad 23$ & $24 \quad 25$ & $26 \quad 27$ & $28 \quad 29$ & $30 \quad 31$ \\
\hline Anode 1 & \multicolumn{5}{|c|}{0} & \multicolumn{4}{|c|}{1} & \multicolumn{4}{|c|}{2} & \multicolumn{4}{|c|}{3} \\
\hline Anode 2 & \multicolumn{3}{|c|}{4} & \multicolumn{2}{|r|}{5} & \multicolumn{2}{|c|}{6} & \multicolumn{2}{|c|}{7} & \multicolumn{2}{|c|}{8} & \multicolumn{2}{|c|}{9} & \multicolumn{2}{|c|}{10} & \multicolumn{2}{|c|}{11} \\
\hline Anode 3 & 12 & 13 & & 14 & 15 & 16 & 17 & 18 & 19 & 20 & 21 & 22 & 23 & 24 & 25 & 26 & 27 \\
\hline Anode 4 & 28 & 29 & & 30 & 31 & 32 & 33 & 34 & 35 & 36 & 37 & 38 & 39 & 40 & 41 & 42 & 43 \\
\hline Anode 5 & 44 & 45 & & 46 & 47 & 48 & 49 & 50 & 51 & 52 & 53 & 54 & 55 & 56 & 57 & 58 & 59 \\
\hline Anode 6 & 60 & 61 & 1 & 62 & 63 & 64 & 65 & 66 & 67 & 68 & 69 & 70 & 71 & 72 & 73 & 74 & 75 \\
\hline Anode 7 & \multicolumn{3}{|c|}{76} & \multicolumn{2}{|c|}{77} & \multicolumn{2}{|c|}{78} & \multicolumn{2}{|c|}{79} & \multicolumn{4}{|c|}{780} & \multicolumn{4}{|c|}{83} \\
\hline Anode 8 & \multicolumn{5}{|c|}{84} & \multicolumn{4}{|c|}{85} & \multicolumn{4}{|c|}{86} & \multicolumn{4}{|c|}{87} \\
\hline
\end{tabular}

b)

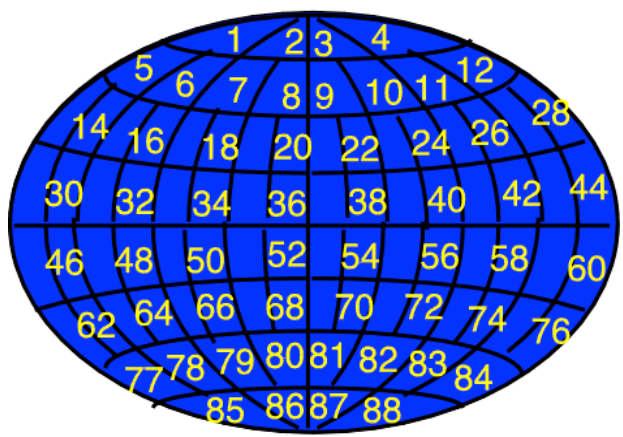

Fig. 8. (a) Angle map showing how the eight position anodes in the instrument are combined with the spacecraft spin to create an 88-angle product. (b) A Mollweide projection showing how the 88 angles cover the full sphere.

\subsection{Relative anode efficiencies}

As discussed above, the change in the efficiency is often not uniform across the MCPs. The efficiencies as determined from the rates, or from pressure balance or density comparisons only give information about the whole side (HS or LS) of the instrument. However, the relative efficiencies of different positions (or anodes) are also important. If an anisotropy is introduced due to non-uniform efficiencies, a calculation of the velocity in the spin-axis direction will result in an anomalous velocity. In order to calculate the velocity vector accurately, the relative calibrations within the instrument must also be corrected.

The method for calculating the relative efficiencies is to normalize the efficiencies at different positions, or anodes, in the instrument by assuming gyrotropy during selected time periods. The selected time periods are either from the outer magnetosphere, where the distribution is normally peaked at 90 degrees, or in the plasma sheet, where the distribution is normally isotropic. This normalization is done using the 3D distribution function products that are included in the science data from the instrument. The data accumulated in the 3-D distribution function products are binned by both species and angle. The eight instrument anodes on each side (HS or LS) give eight $22.5^{\circ}$ polar angles, as described above. The azimuthal angle, in the spin plane, is determined by syncing the data accumulation to the spacecraft spin. The full spin is divided into 16 sectors of $22.5^{\circ}$. Figure 8 a shows how the eight polar angles and 16 azimuthal angles are combined to give 88 total angles. Figure $8 \mathrm{~b}$ shows how these 88 angles cover the sphere in a Mollweide projection. Depending on the magnetic field orientation, one anode (corresponding to one row in Fig. 8a) can cover a range of pitch angles during a spacecraft spin. The flux is calculated for each of the 88 angles, and is plotted at the pitch angle determined for that position. Each anode contributes 4 to 16 individual points on the pitch angle plot. Then each anode is individually adjusted so that the flux is the same at a given pitch angle.

Figures 9-11 illustrate an example from a time when the spacecraft are in the plasma sheet. Figure 9 shows the pitch angle distribution for $\mathrm{H}^{+}$at 11 different energies. In each panel, there is one point for each of the 88 angles in the 3$\mathrm{D}$ distribution. The different colors/symbols indicate which anode was used. A fit is performed to the points around $90^{\circ}$ $\left(90^{\circ} \pm 45^{\circ}\right)$ in order to determine the average pitch angle distribution, as shown with a black line in Fig. 9. Then a normalization factor is determined for each anode at each energy that will make the flux measured by that anode, over the range of pitch angles that it covers, best match the average fit. In this case, the flux of anode 8 (red) is systematically high, while the flux for anode 5 (greenish yellow) is low. The resulting normalized pitch angle plots are shown in Fig. 10. 


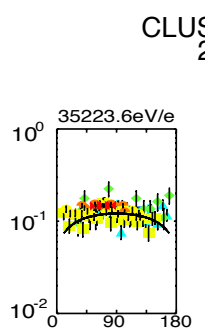

USTER/CODIF Product 13 Satellite 4 2007-08-21/14:30:00 - 23:00:03 High Side
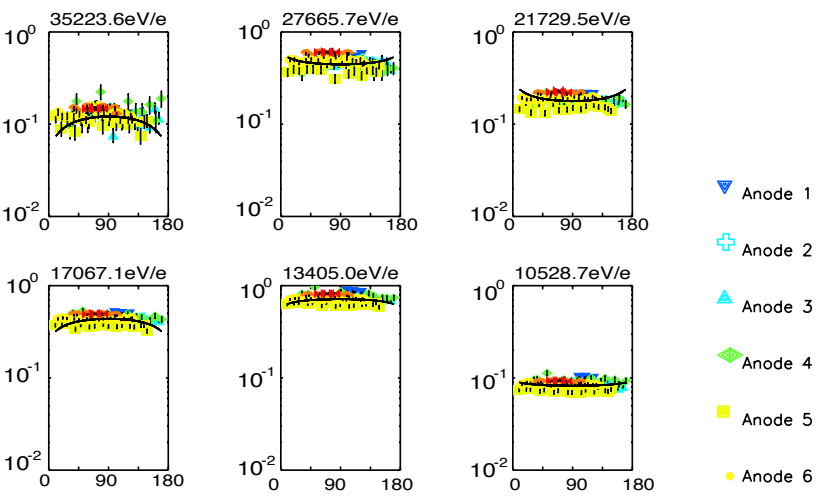

↔ Anode 2

Anode 3

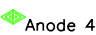

Anode 5

Anode 6
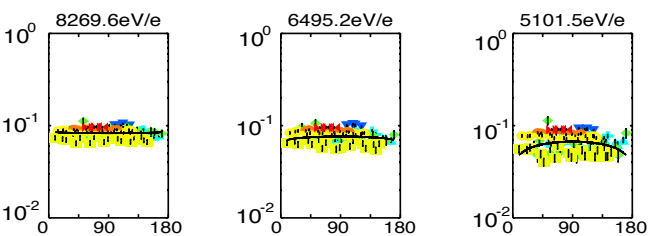

Anode 7

Anode 8
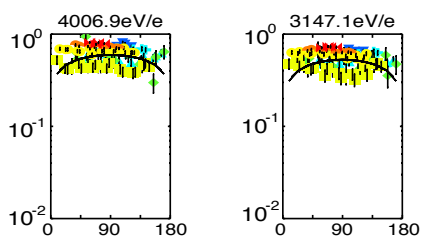

Fig. 9. The flux as a function of pitch angle for individual anodes on the CODIF high side. Each panel shows a different energy channel, ranging from $35 \mathrm{keV}$ (top left panel) to $3 \mathrm{keV}$ (bottom right panel).

Now, for each energy and pitch angle, all anodes give the same flux. Then, the adjustment parameters determined for each anode and energy are used to redefine the calibration curves. The final result is shown in Fig. 11. The top panel shows the original curves used to make the first pitch angle plot, and the bottom panel shows the revised curves. To make the revised curves, the points for each anode at each energy where there are sufficient counts to make a pitch angle fit are shifted based on the normalization factor. Then additional points are added at 60 and $14 \mathrm{keV}$ to force the fit to follow the same trend as the data at the outer limits of the instrument energy range. Then a fit is done to this new set of points to generate the curve. This procedure is performed about once per month, and more often if the distributions show a significant change.

After the new files with the relative anode efficiencies have been determined, the efficiencies are checked by calculating the average velocities in the plasma sheet. Figure 12 shows a comparison of orbit averages of the density and the three components of the velocity for CODIF on SC4, and HIA on $\mathrm{SC} 1$ and SC3, in GSE coordinates. The $x$ and $y$ coordinates are approximately in the spin plane, and so the relative anode efficiency does not significantly affect these velocities. The
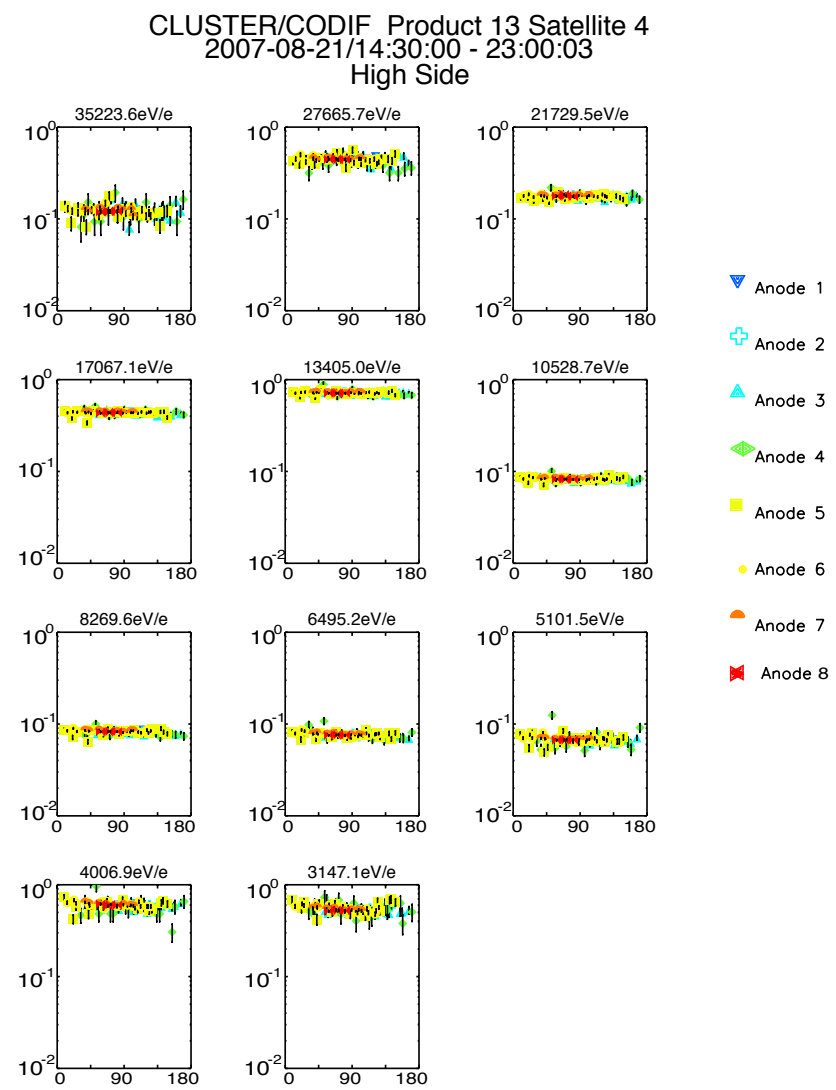

Fig. 10. The flux as a function of pitch angle for individual anodes on the CODIF high side after each individual anode has been normalized.

$z$ coordinate is almost along the spin plane, so any discrepancies in the relative anode efficiencies are observed here. It is expected that the average velocity in $z$ should be 0 . The relative anode efficiencies are adjusted to keep the error to less than $15 \mathrm{~km} \mathrm{~s}^{-1}$.

\section{$3 \mathrm{O}^{+}$calibration}

$\mathrm{O}^{+}$is the second most abundant species in the magnetosphere, and is the key tracer for ionospheric input. Thus tracking the $\mathrm{O}^{+}$efficiencies is critical for measuring the importance of the ionosphere as a source of plasma in the magnetosphere over the mission.

\subsection{Relative anode efficiencies}

The relative anode efficiencies for $\mathrm{O}^{+}$can be determined in exactly the same way as they are determined for $\mathrm{H}^{+}$. Because the method uses data that are classified by species in the instrument, it does not require time periods where $\mathrm{O}^{+}$is dominant. We still need to identify time periods when there is a significant flux of $\mathrm{O}^{+}$, so that we have sufficient statistics to compare the different anodes, but $\mathrm{H}^{+}$can still be the 


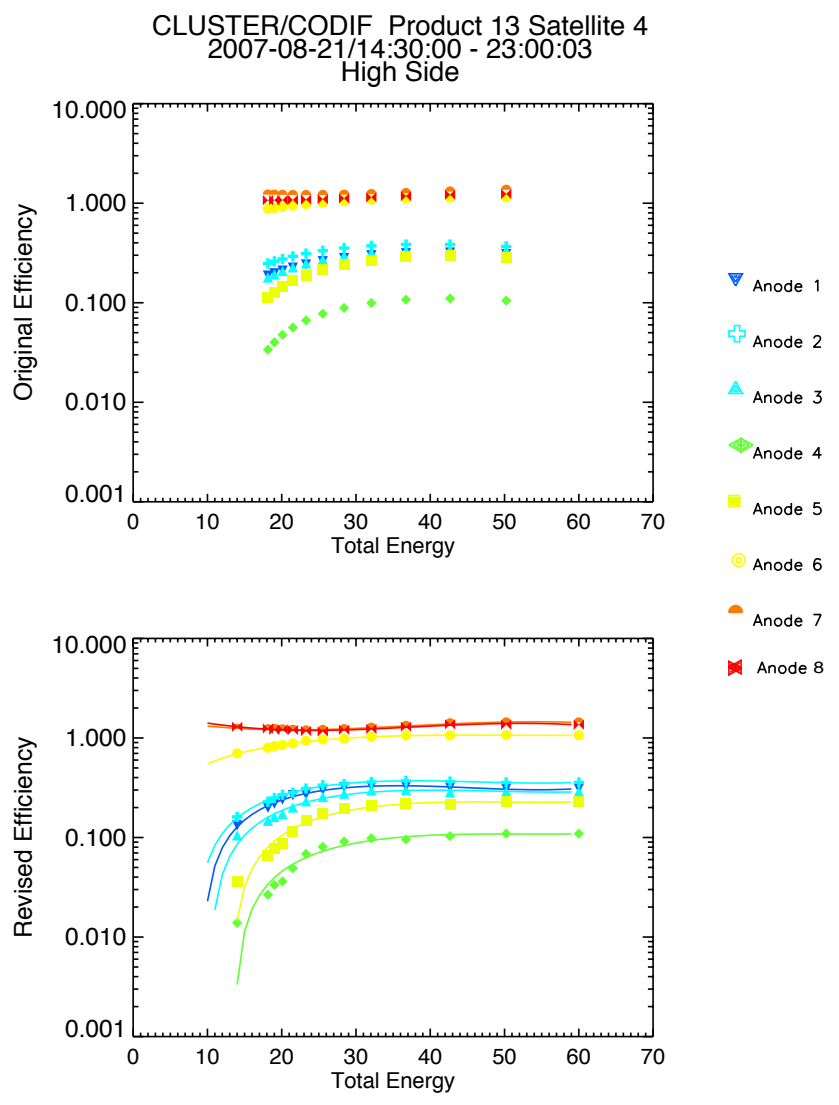

Fig. 11. The top panel shows the relative anode efficiency as a function of energy for each of the eight anodes on the CODIF high side. The bottom panel shows revised anode efficiencies, using the normalization factors determined assuming a gyrotropic distribution.

dominant ion. An example of an $\mathrm{O}^{+}$relative calibration is shown in McFadden et al. (2007).

\subsection{Total efficiencies}

Determining $\mathrm{O}^{+}$total efficiencies in-flight is difficult because all the techniques applied for $\mathrm{H}^{+}$are only useful for the dominant species. In order to use the engineering rate techniques, we need to find time periods when $\mathrm{O}^{+}$is actually the dominant species, at least at a particular energy per charge, so that the start, stop, and coincidence rates that we measure are due to $\mathrm{O}^{+}$. The only time periods that we have found to do this reliably are times when the spacecraft is over the polar cap and in the lobes. In these regions, we sometimes observe narrow energy "beams" of $\mathrm{O}^{+}$. The origin of these beams is outflow from the cusp. Because the ions move up along the field lines, as the field line is convected into the tail, the original particle distribution, which can be broad in energy, becomes separated by velocity, because the higher velocity ions are moving further along the field line than the lower velocity ions. This is referred to as the "velocity filter effect". Since $\mathrm{O}^{+}$and $\mathrm{H}^{+}$at the same velocity are separated

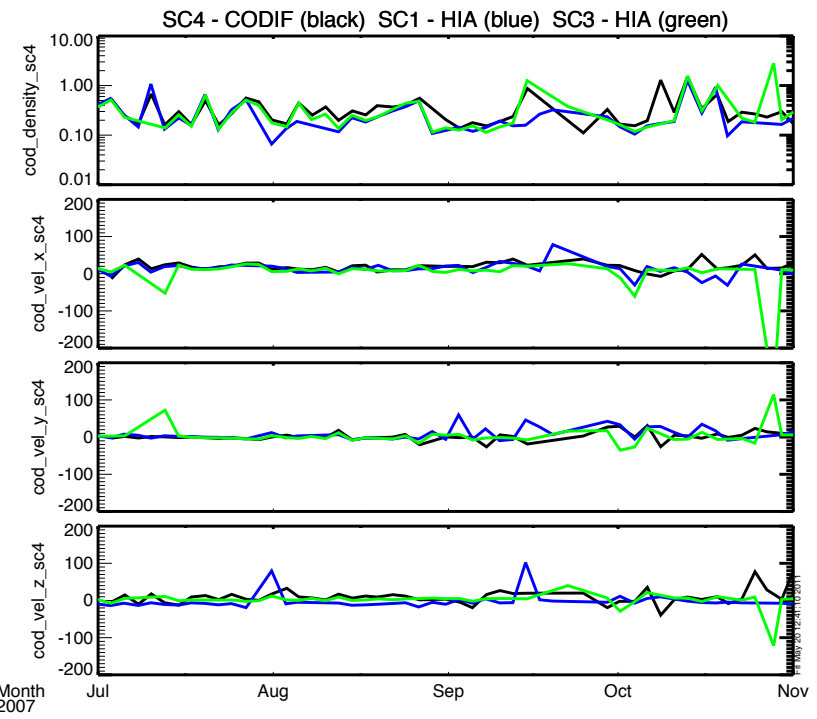

Fig. 12. The average densities and velocities in the plasma sheet from CODIF, on S/C 4, and from HIA on S/C 1 and S/C 3.

in energy per charge by a factor of 16 , the $\mathrm{O}^{+}$beam is observed at a distinctly different energy step in the instrument than the $\mathrm{H}^{+}$beam. Thus we can use the energy steps where the $\mathrm{O}^{+}$is observed to determine the $\mathrm{O}^{+}$efficiency. Because the $\mathrm{O}^{+}$beams are generally field-aligned, they are only observed in one or two anodes at a time. Thus we can obtain the total efficiencies for a particular anode. We then use the data from the relative anode $\mathrm{O}^{+}$calibration to determine an overall change to the total efficiency.

An example of these $\mathrm{O}^{+}$beams is shown in Fig. 13. During this time period on 21 December 2002, a strong $\mathrm{O}^{+}$beam is clearly observed as the spacecraft approaches the cusp. Figure 13 shows the $\mathrm{O}^{+}$energy spectra for the 8 individual anodes on the HS. In this case, the beam is initially observed in anode 1 . It then moves to anode 2 , and then into 3 . The beams are observed for more than an hour in each of the anodes 1 and 2. Thus we can accumulate sufficient statistics to use the rate data to calculate the $\mathrm{O}^{+}$efficiencies. For 20012004, we used this method to determine the $\mathrm{O}^{+}$calibration for $\mathrm{SC} / 1$. The final $\mathrm{S} / \mathrm{C} 1 \mathrm{O}^{+}$normalized total efficiencies derived using this method are shown in Fig. 14. The $\mathrm{H}^{+}$normalized total efficiency (green line) is shown for comparison. The calibrations for S/C 3 and S/C 4 were then done by comparison with S/C 1. Stable time periods were chosen when the three spacecraft would be expected to observe the same flux, and the efficiencies were adjusted to bring the $\mathrm{O}^{+}$fluxes on S/C 3 and S/C 4 to the same level as S/C 1 . About one time period per month is used for this adjustment.

After 2004, the same method was used, using beams observed by S/C 4 . However, this method was only useful through 2006. After 2006, the flux of $\mathrm{O}^{+}$in these regions decreased significantly, because there is less ionospheric outflow during solar minimum (Yau and André, 


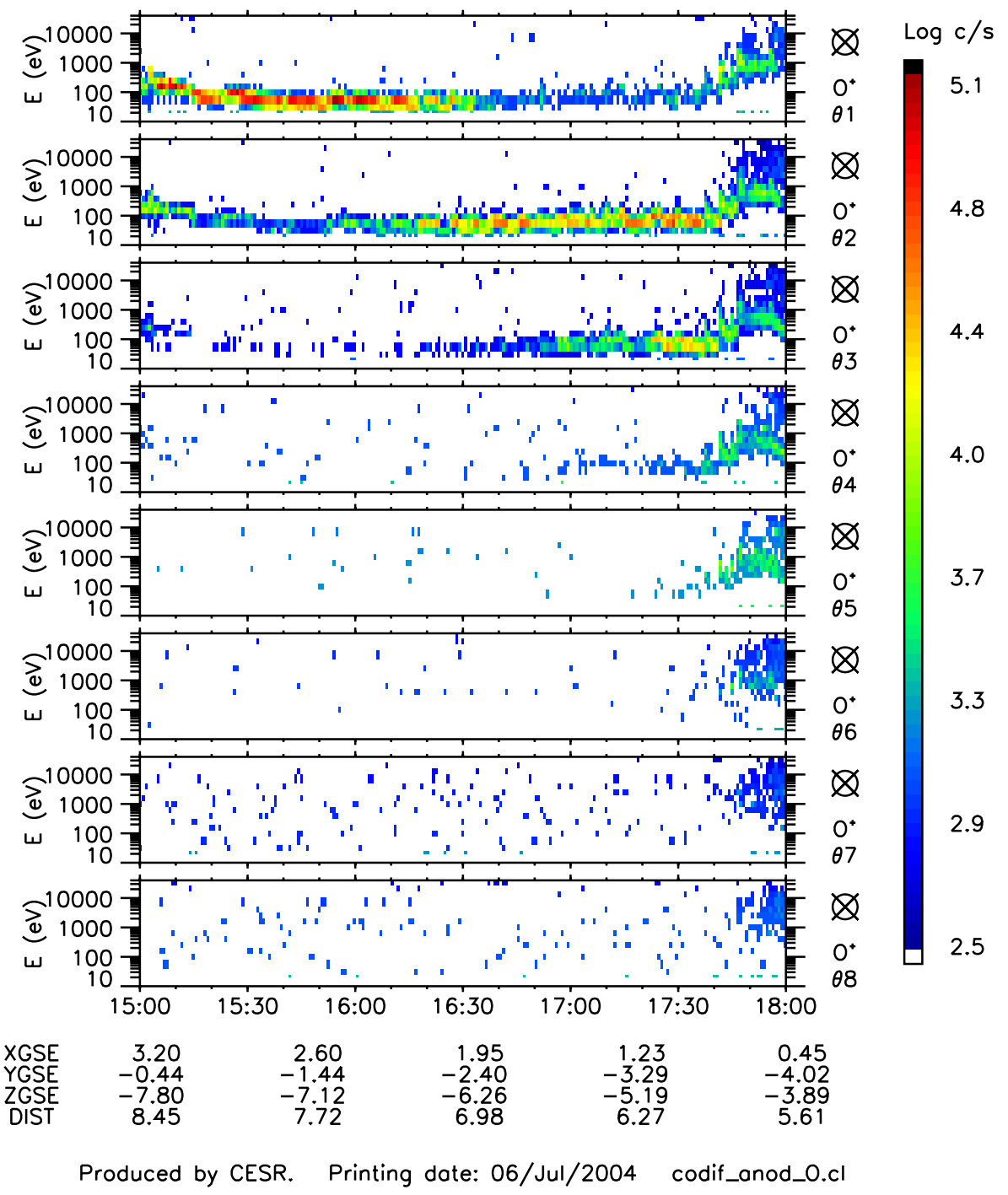

Fig. 13. Example of $\mathrm{O}^{+}$beams observed on 21 December 2002, on SC 1, as the spacecraft approaches the cusp. Each panel shows the $\mathrm{O}^{+}$ energy spectrum for one position anode in the instrument. In this case, the beam is first predominantly in anode 1 , and then moves to anode 2 , and then 3 .

1997). Therefore, we were no longer able to do this measurement. Now that the solar cycle is approaching solar maximum, we again observe $\mathrm{O}^{+}$beams. Using new time periods, we will again be able to measure the total efficiency, and adjust the $\mathrm{O}^{+}$efficiency values. At this point, the $\mathrm{O}^{+}$efficiencies are considered valid through 2006 , and are estimated after that. A new release of calibration files will update the $\mathrm{O}^{+}$ total efficiency values.

\section{Other species}

Because the $\mathrm{He}^{++}$suffers from significant contamination from $\mathrm{H}^{+}$, as discussed in the companion paper by Mouikis et al. (2013), no separate calibration for $\mathrm{He}^{++}$has been determined. $\mathrm{He}^{++}$fluxes are calculated using the initial cali- bration curves determined pre-launch, and with the total efficiencies following the $\mathrm{H}^{+}$changes.

$\mathrm{He}^{+}$is also difficult to calibrate because there are not many time periods when its flux is significant. From the preflight calibrations, the $\mathrm{He}^{+}$efficiency was found to be very similar to the $\mathrm{O}^{+}$efficiency, except at the low energies, where $\mathrm{O}^{+}$has a lower efficiency due to the greater scattering in the foil. Thus we have used the energy dependence for $\mathrm{He}^{+}$that we established pre-flight, but then assumed that the total efficiency changes with time following the $\mathrm{O}^{+}$efficiency.

\section{Conclusions}

Using a variety of methods, we have determined the calibrations of $\mathrm{H}^{+}$through the duration of the mission. We were 


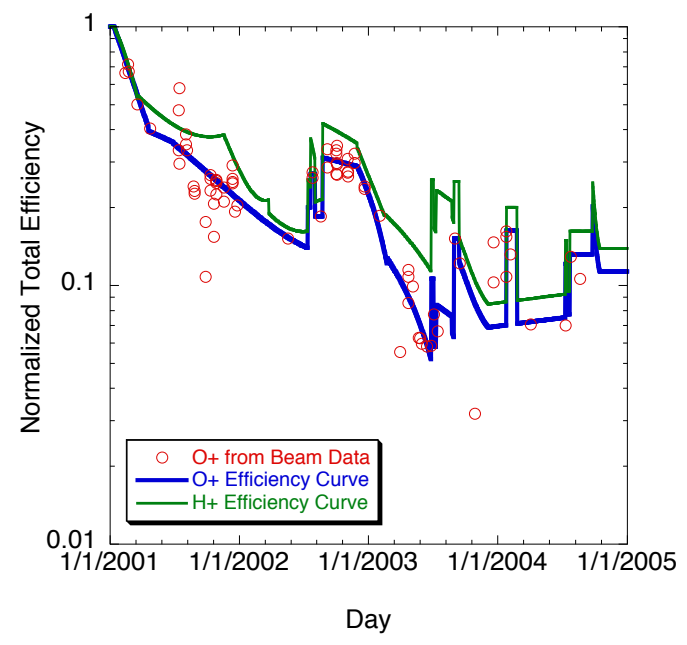

Fig. 14. $\mathrm{O}^{+}$normalized total efficiency derived using the $\mathrm{O}^{+}$beam data for S/C 1. The final curve used for the efficiency is shown with the blue line, and the $\mathrm{H}^{+}$efficiency is shown in green, for comparison.

able to calibrate the total efficiencies of $\mathrm{O}^{+}$successfully through 2006 using the $\mathrm{O}^{+}$"beams" observed in the lobe. Due to the diminished ionospheric outflow during the solar minimum, we have only been able to determine the relative anode calibrations after 2006, while the total efficiency calibration is only an estimate. However, now that the mission is again approaching solar maximum, the beams are observed again, and we will use them to confirm the $\mathrm{O}^{+}$calibration. After the last MCP voltage increase in 2009, the efficiencies have remained relatively stable for more than three years. We anticipate that CODIF on S/C 4 will continue returning data with reasonable efficiencies through the extended mission.

Acknowledgements. We are grateful to the many engineers and scientists from UNH, MPE, CESR, MPS, IFSI, IRF, UCB and UW who made the development of the CIS instrument possible. The calibration work at UNH was supported by NASA under grant NNX11AB65G. We also thank the other Cluster teams, in particular the WHISPER team (Principle Investigator, Jean Louis Rauch; former Principle Investigators, Pierrette M. E. Decreau, Jean Gabriel Trotigen; and Gabor Fascko), the PEACE team (Principle Investigator, A. Fazakerly), and the MAG team (Principle Investigator, E. Lucek) for making their data available for the cross-calibration work.

Edited by: M. Genzer

\section{References}

Allegrini, F., Wimmer-Schweingruber, R. F., Wurz, P., and Bochsler, P.: Determination of low-energy ion-induced electron yields from thin carbon foils, Nucl. Instrum. Meth. Phys. Res. B, 211, 487-494, doi:10.1016/S0168-583X(03)01705-1, 2003.
Allegrini, F., McComas, D. J., Young, D. T., Berthelier, J. J., Covinhes, J., Illiano, J. M., Riou, J. F., Funsten, H. O., and Harper, R. W.: Energy loss of 1-50 keV H, He, C, N, O, Ne, and Ar ions transmitted through thin carbon foils, Rev. Sci. Instrum., 77, 4501, doi:10.1063/1.2185490, 2006.

Blagau, A., Dandouras, I., Barthe, A., Brunato, S., Facskó, G., and Constantinescu, V.: In-flight calibration of Hot Ion Analyser onboard Cluster, Geosci. Instrum. Method. Data Syst. Discuss., 3, 407-435, doi:10.5194/gid-3-407-2013, 2013.

Décréau, P. M. E., Fergeau, P., Krasnoselskikh, V., Le Guirriec, E., Lévêque, M., Martin, Ph., Randriamboarison, O., Rauch, J. L., Sené, F. X., Séran, H. C., Trotignon, J. G., Canu, P., Cornilleau, N., de Féraudy, H., Alleyne, H., Yearby, K., Mögensen, P. B., Gustafsson, G., André, M., Gurnett, D. C., Darrouzet, F., Lemaire, J., Harvey, C. C., Travnicek, P., and Whisper experimenters (Table 1): Early results from the Whisper instrument on Cluster: an overview, Ann. Geophys., 19, 1241-1258, doi:10.5194/angeo-19-1241-2001, 2001.

Drake, V. A., Eparvier, F. G., McClintock, W. E., Woods, T. N., Ucker, G. J., and Hill, C.: Microchannel plate performance and life-test results for the TIMED Solar EUV experiment, Proc. SPIE, 3445, 603-614, 1998.

Fairfield, D. H., Lepping, R. P., Hones, E. W. J., Bame, S. J., and Asbridge, J. R.: Simultaneous measurements of magnetotail dynamics by IMP spacecraft, J. Geophys. Res., 86, 1396-1414, doi:10.1029/JA086iA03p01396, 1981.

Gonin, M., Buergi, A., Oetliker, M., and Bochsler, P.: Interaction of solar wind ions with thin carbon foils: Calibration of timeof-flight spectrometers, in: ESA, Proceedings of the First SOHO Workshop: Coronal Streamers, Coronal Loops, and Coronal and Solar Wind Composition (SEE N93-31343 12-92), 348, 381384, 1992.

Johnstone, A. D., Alsop, C., Burge, S., Carter, P. J., Coates, A. J., Coker, A. J., Fazakerley, A. N., Grande, M., Gowen, R. A., Gurgiolo, C., Hancock, B. K., Narheim, B., Preece, A., Sheather, P. H., Winningham, J. D., and Woodliffe, R. D.: Peace: a Plasma Electron and Current Experiment, Space Sci. Rev., 79, 351-398, doi:10.1023/A:1004938001388, 1997.

Kishimoto, N., Nagamine, M., Inami, K., Enari, Y., and Ohshima, T.: Lifetime of MCP-PMT, Nucl. Instrum. Meth. Phys. Res. A, 564, 204-211, doi:10.1016/j.nima.2006.04.089, 2006.

Kistler, L. M.: Cluster CODIF Calibration Report, Part I, Ion Efficiency Analysis, available at: http://caa.estec.esa.int/documents/ CLUSTER_CODIFEff.pdf (last access: 25 April 2013), 2000a.

Kistler, L. M.: Cluster CODIF Calibration Report, Part II, Geometric Factor, Energy and Angle Response, available at: http:// caa.estec.esa.int/documents/CODIF_ESAAlpha.pdf (last access: 25 April 2013), 2000b.

Kistler, L. M.: Cluster CODIF Calibration Report, Part III, TOF Peak Analysis, available at: http://caa.estec.esa.int/documents/ CODIF_CalTOFv2.pdf (last access: 25 April 2013), 2000c.

McFadden, J. P., Evans, D. S., Kasprzak, W. T., Brace, L. H., Chornay, D. J., Coates, A. J., Dichter, B. K. H. W. R., Holeman, E., Kadinsky-Cade, K., Kasper, J. C., Kataria, D., Kistler, L., Larson, D., Lazarus, A. J. M. F., Mukai, T., Ogilvie, K. W., Paschmann, G., Rich, F., Saito, Y., Sudder, J. D., Steinberg, J. T., Wuest, M., and Wurz, P.: In-Flight Instrument Calibration and Performance Verification, Calibr. Part. Instrum. Space Phys., 7, 277385, 2007. 
Möbius, E., Kistler, L. M., Popecki, M. A., Crocker, K. C., Granoff, M., Jiang, Y., Sartori, E., Ye, V., Rème, H., Sauvaud, J. A., Cros, A., Aoustin, C., Camus, T., Medale, J. L., Rouzaud, J., Carlson, C. W., McFadden, J. P., Curtis, D., Heetder, H., Croyle, J., Ingraham, C., Klecker, B., Hovestadt, D., Ertl, M., Eberl, F., Kästle, H., Künneth, E., Laeverenz, P., Seidenschwang, E., Shelley, E. G., Klumpar, D. M., Hertzberg, E., Parks, G. K., McCarthy, M., Korth, A., Rosenbauer, H., Gräve, B., Eliasson, L., Olsen, S., Balsiger, H., Schwab, U., and Steinacher, M.: The 3-D Plasma Distribution Function Analyzers with Time-of-Flight Mass Discrimination for Cluster, FAST, and Equator-S, in: Measurement Techniques in Space Plasmas:Particles, edited by: Pfaff, R. F., Borovsky, J. E., and Young, D. T., American Geophysical Union, Washington, D. C., doi:10.1029/GM102p0243, 2013.

Mouikis, C. G., Kistler, L. M., Wang, G., and Liu, Y.: Background subtraction for the Cluster/CODIF plasma ion mass spectrometer, Geosci. Instrum. Method. Data Syst. Discuss., 3, 567-589, doi:10.5194/gid-3-567-2013, 2013.

Rème, H., Aoustin, C., Bosqued, J. M., Dandouras, I., Lavraud, B., Sauvaud, J. A., Barthe, A., Bouyssou, J., Camus, Th., Coeur-Joly, O., Cros, A., Cuvilo, J., Ducay, F., Garbarowitz, Y., Medale, J. L., Penou, E., Perrier, H., Romefort, D., Rouzaud, J., Vallat, C., Alcaydé, D., Jacquey, C., Mazelle, C., d'Uston, C., Möbius, E., Kistler, L. M., Crocker, K., Granoff, M., Mouikis, C., Popecki, M., Vosbury, M., Klecker, B., Hovestadt, D., Kucharek, H., Kuenneth, E., Paschmann, G., Scholer, M., Sckopke, N., Seidenschwang, E., Carlson, C. W., Curtis, D. W., Ingraham, C., Lin, R. P., McFadden, J. P., Parks, G. K., Phan, T., Formisano, V., Amata, E., Bavassano-Cattaneo, M. B., Baldetti, P., Bruno, R., Chionchio, G., Di Lellis, A., Marcucci, M. F., Pallocchia, G., Korth, A., Daly, P. W., Graeve, B., Rosenbauer, H., Vasyliunas, V., McCarthy, M., Wilber, M., Eliasson, L., Lundin, R., Olsen, S., Shelley, E. G., Fuselier, S., Ghielmetti, A. G., Lennartsson, W., Escoubet, C. P., Balsiger, H., Friedel, R., Cao, J.-B., Kovrazhkin, R. A., Papamastorakis, I., Pellat, R., Scudder, J., and Sonnerup, B.: First multispacecraft ion measurements in and near the Earth's magnetosphere with the identical Cluster ion spectrometry (CIS) experiment, Ann. Geophys., 19, 1303-1354, doi:10.5194/angeo19-1303-2001, 2001.
Ritzau, S. M. and Baragiola, R. A.: Electron emission from carbon foils induced by keV ions, Phys. Rev. B, 58, 2529-2538, doi:10.1103/PhysRevB.58.2529, 1998.

Sandel, B. R., Broadfoot, A. L., and Shemansky, D. E.: Microchannel plate life tests, Appl. Optics, 16, 1435-1437, doi:10.1364/AO.16.001435, 1977.

Trotignon, J. G., Décréau, P. M. E., Rauch, J. L., Vallières, X., Rochel, A., Kougblénou, S., Lointier, F., Facskó, G., Canu, P., Darrouzet, F., and Masson, A.: The WHISPER Relaxation Sounder and the Cluster Active Archive, in: The Cluster Active Archive, C. P. Astrophysics and Space Science Proceedings, edited by: Laakso, H., Taylor, M. G. T. T., and Escoubet, Springer, Berlin, 185-208, doi:10.1007/978-90-481-3499-1_12, 2010.

Yau, A. W. and André, M.: Sources of Ion Outflow in the High Latitude Ionosphere, Space Sci. Rev., 80, 1-25, doi:10.1023/A:1004947203046, 1997. 